\title{
THE ABSOLUTE CONFIGURATION OF DECILONITROSE, A SUGAR COMPONENT OF DECILORUBICIN, IS UNDOUBTEDLY 2,3,6-TRIDEOXY-3-C-METHYL-3-NITRO-L-RIBO-HEXOPYRANOSE
}

\author{
Yoshio Nishimura, KiYoto IsHI and Shinichi Kondo \\ Institute of Microbial Chemistry, \\ 3-14-23 Kamiosaki, Shinagawa-ku, Tokyo 141, Japan \\ (Received for publication July 21, 1989)
}

\begin{abstract}
The absolute structure of decilonitrose, a sugar component of an antitumor antibiotic decilorubicin was decided to be 2,3,6-trideoxy-3-C-methyl-3-nitro-L-ribo-hexopyranose by synthesis of its methyl $\beta$-glycoside starting from L-rhamnose through the 3-ulose. In the synthetic route, any configurational ambiguities do not exist.
\end{abstract}

Decilonitrose (1), a new nitro branched-chain sugar, was observed as a sugar component in decilorubicin (2). ${ }^{1)}$ Methyl decilonitroside (3), $[\alpha]_{\mathrm{D}}-13^{\circ}\left(\mathrm{CHCl}_{3}\right)$, was obtained by methanolysis of 2 . In the previous communication, ${ }^{2)}$ we reported its total synthesis and absolute configuration as methyl 2,3,6-trideoxy-3$C$-methyl-3-nitro- $\beta$-L-ribo-hexopyranoside. Decilonitrose was also found in arugomycin (4), and its methanolysis afforded $3,[\alpha]_{\mathrm{D}}-10^{\circ}\left(\mathrm{CHCl}_{3}\right) .^{3,4)}$ In the recent article, ${ }^{5)} \mathrm{ZEECK}$ et al. have reported the isolation of a methyl glycoside of a nitro branched-chain sugar, $[\alpha]_{\mathrm{D}}-17.5^{\circ}\left(\mathrm{CHCl}_{3}\right)$, supposed to be 3 by methanolysis of viriplanin $\mathrm{D}$, a photooxidation product of raw viriplanin. They concluded its absolute configuration as $\beta$-D-series by a misunderstanding application of HuDsoN's isorotation rule ${ }^{6)}$ compared with methyl $\alpha$-D-decilonitroside, $[\alpha]_{\mathrm{D}}+141.8^{\circ}\left(\mathrm{CHCl}_{3}\right)$ (synthetic $\alpha$-L-decilonitroside, ${ }^{7)}[\alpha]_{\mathrm{D}}-172^{\circ} \pm 3^{\circ}$ $\left(\mathrm{CHCl}_{3}\right)$ ). Their methyl $\alpha$-D-glycoside was obtained by successive sequences of hydrazine reduction, methanolysis and peracid oxidation of viriplanin $\mathrm{A}^{5}$ whose structure was not clarified. From these results they pointed out our methyl decilonitroside to be $\mathrm{D}$-series.

In this paper, we want to verify our conclusion to be absolutely correct with the full experimental details and discussion. ${ }^{8,9), \dagger}$ Key stages in our strategy followed Yoshimura's excellent procedure for the synthesis of evernitrose (5) and 3-epi-evernitrose (6) involved subsequent reactions of cyanomesylation of 3-ulose (11), reduction into spiro-aziridine, hydrogenolysis of the aziridine ring and oxidation to the nitro group $^{10)}$ (Scheme 1). The synthesis of our key intermediate 11 began with methyl 2,6-dideoxy- $\beta$-L-arabinohexopyranoside (7) which was synthesized starting from L-rhamnose. ${ }^{11), \dagger \dagger}$ Selective acylation of 7 with benzoyl chloride in pyridine afforded 3-O-benzoate 8 in a yield of $71 \%$. Compound 8 was converted to 4- $O$-benzyl ether 10 by benzylation (benzyl chloride, $\mathrm{NaH}, N, N$-dimethylformamide; $9,65 \%$ yield) followed by deacylation (sodium methoxide, methanol, $85 \%$ yield). Oxidation of 10 with $\mathrm{CrO}_{3}$-pyridine in dichloromethane afforded 11 in a yield of $73 \%$. Cyanomesylation of 11 was carried out by successive

The total synthesis and absolute configuration of 3 was presented with the full experimental details on the Annual Meeting of the Agricultural Chemical Society of Japan in $1983^{8)}$ and also described in detail in the Doctor Thesis by K. IsHu (University of Tokyo, 1983). ${ }^{\text {) }}$

it Compound 7 was also prepared by the following method: Treatment of 1,5-anhydro-3,4-di- $O$-acetyl-2,6-dideoxyt-arabino-hex-1-enitol (3,4-di- $O$-acetylrhamnal) with $\mathrm{Br}_{2}$ in dichloromethane, and subsequent reaction with methanol and $\mathrm{Ag}_{2} \mathrm{CO}_{3}$, followed by hydrogenation with $\mathrm{Pd}-\mathrm{C}$ in a mixture of methanol, water and triethylamine $(5: 4: 1)$ under hydrogen at $3.5 \mathrm{~kg} / \mathrm{cm}^{2}$ afforded 7 (49\% yield) and its $\alpha$-anomer $(16 \%$ yield). 
reaction of $\mathrm{HCN}$ and methanesulfonyl chloride in pyridine to give the 3-cyano-3-O-mesyl derivatives having L-arabino (12, 42\% yield) and L-ribo $(\mathbf{1 3}, 23 \%$ yield) configurations. The stereochemistries of 12 and 13 were established at the later stage. Compounds 12 and 13 were transformed into the spiro-aziridine derivatives 14 and 15 by reduction with lithium aluminum hydride in $66 \%$ and $50 \%$ yields, respectively. Catalytic hydrogenation of $\mathbf{1 4}$ and $\mathbf{1 5}$ with Raney nickel catalyst gave the 3-amino-3methyl derivatives having L-ribo $(\mathbf{1 6}, 83 \%$ yield, $\left.[\alpha]_{\mathrm{D}}+45.6^{\circ}\left(\mathrm{CHCl}_{3}\right)\right)$ and L-arabino $(17,86 \%$ yield,

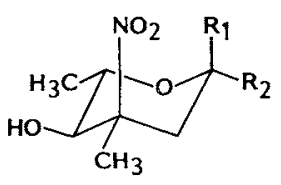

Decilonitrose (1) $\mathrm{R}_{1}, \mathrm{R}_{2}=\mathrm{H}, \mathrm{OH}$ Methyl decilonitroside (3) $\quad \mathrm{R}_{1}=\mathrm{H} \quad \mathrm{R}_{2}=\mathrm{OCH}_{3}$

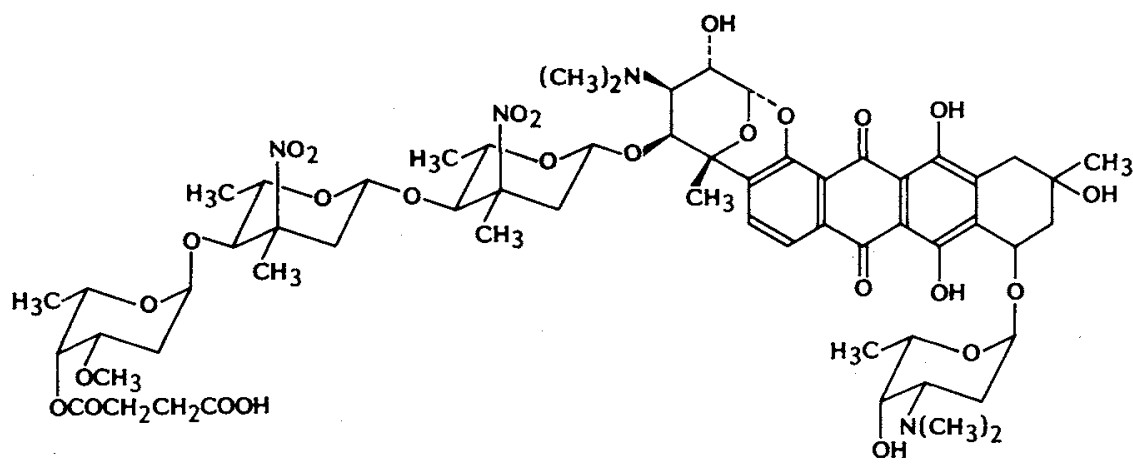

Decilorubicin (2)

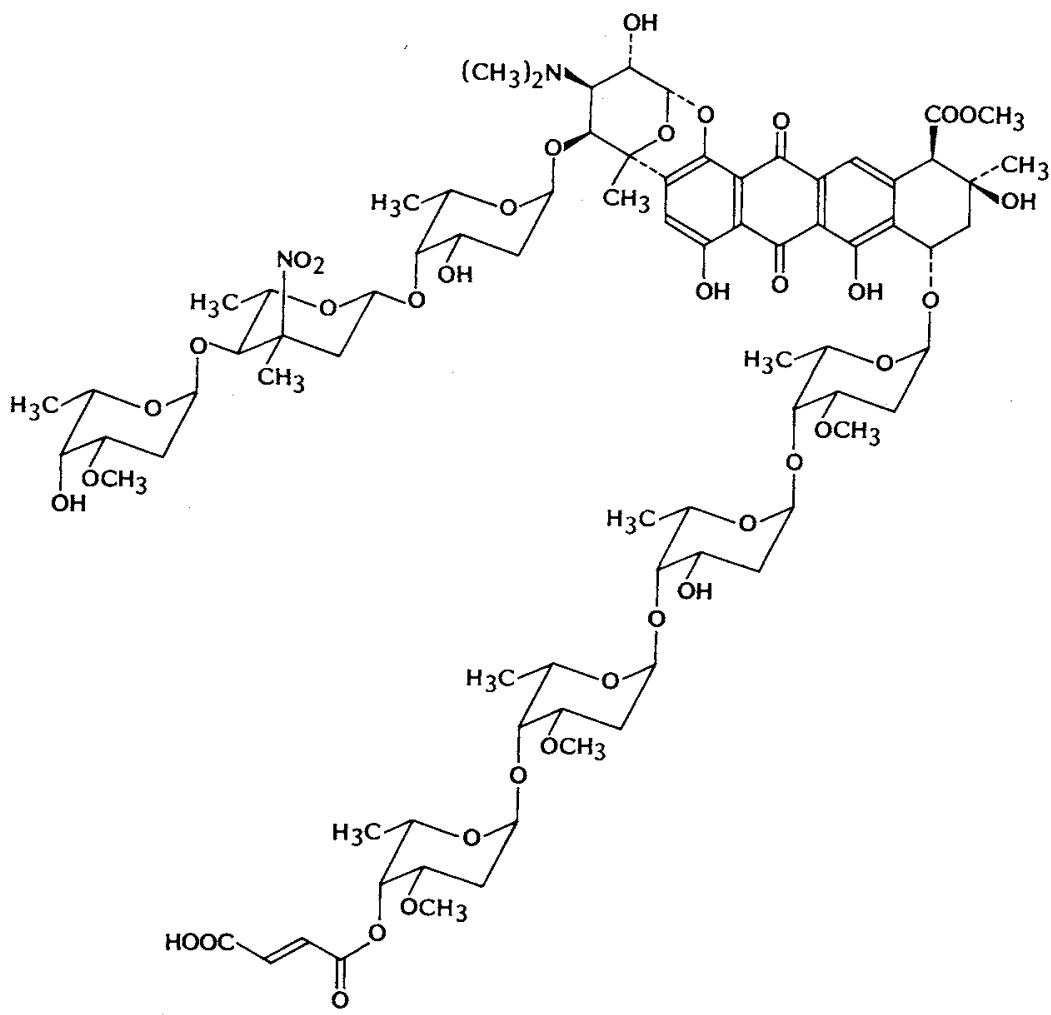

Arugomycin $^{3,4)}(4)$ 
$\left.[\alpha]_{\mathrm{D}}+33.3^{\circ}\left(\mathrm{CHCl}_{3}\right)\right)$ configurations.

The absolute configurations of $\mathbf{1 6}$ and $\mathbf{1 7}$ were established by application of the TACu method: ${ }^{12)}$ 16 showed positive contribution $\left(\Delta[\mathrm{M}]_{436 \text { (TACu) }}\right.$ $\left.+525^{\circ}\right)$ and 17 showed negative $\left(\Delta[\mathrm{M}]_{436 \text { (TACu) }}\right.$ $-621^{\circ}$ ), clearly indicative of L-ribo and L-arabino configurations in 16 and 17 , respectively. Com-

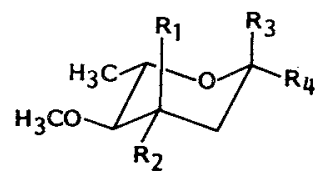

$5 \quad \mathrm{R}_{1}=\mathrm{CH}_{3} \quad \mathrm{R}_{2}=\mathrm{NO}_{2} \quad \mathrm{R}_{3}, \mathrm{R}_{4}=\mathrm{H}, \mathrm{OH}$

$6 \quad \mathrm{R}_{1}=\mathrm{NO}_{2} \quad \mathrm{R}_{2}=\mathrm{CH}_{3} \quad \mathrm{R}_{3}, \mathrm{R}_{4}=\mathrm{H}, \mathrm{OH}$

$21 \quad \mathrm{R}_{1}=\mathrm{NO}_{2} \quad \mathrm{R}_{2}=\mathrm{CH}_{3} \quad \mathrm{R}_{3}=\mathrm{H} \quad \mathrm{R}_{4}=\mathrm{OCH}_{3}$

$22 \quad \mathrm{R}_{1}=\mathrm{CH}_{3} \quad \mathrm{R}_{2}=\mathrm{NO}_{2} \quad \mathrm{R}_{3}=\mathrm{H} \quad \mathrm{R}_{4}=\mathrm{OCH}_{3}$

pounds 16 and 17 were also characterized as the $N$-acetyl derivatives $18\left([\alpha]_{\mathrm{D}}+11^{\circ}\left(\mathrm{CHCl}_{3}\right)\right)$ and 19 $\left([\alpha]_{\mathrm{D}}+12.4^{\circ}\left(\mathrm{CHCl}_{3}\right)\right)$, respectively. In ${ }^{13} \mathrm{C}$ NMR spectra of 18 and 19 , the former showed a singlet assigned to 3-C-methyl at $\delta 24.8$ and the latter showed it at $\delta 18.3$, clearly indicative an equatorial methyl and an axial one in $\mathbf{1 8}$ and $\mathbf{1 9}$, respectively, in agreement with SATo's empirical rule. ${ }^{13)}$ Thus, the absolute configuration of $\mathbf{1 6}$ and $\mathbf{1 7}$ have been determined, and $\mathbf{1 6}$ is the desired compound for synthesis of 1 .

Oxidation of 16 with $m$-chloroperbenzoic acid in acetonitrile gave 3 ( $29 \%$ yield), which was identical with the natural 3 derived from decilorubicin in all respects. Therefore, the structure of $\mathbf{3}$ was absolutely clarified to be methyl 2,3,6-trideoxy-3-C-methyl-3-nitro- $\beta$-L-ribo-hexopyranoside. The $\beta$-L-arabino isomer (20, a colorless foam, $[\alpha]_{\mathrm{D}}+41.7^{\circ}\left(\mathrm{CHCl}_{3}\right)$ ) was also synthesized from 17 in $62 \%$ yield. The ${ }^{13} \mathrm{C}$ NMR chemical shift of the 3-equatorial methyl carbon in $3(\delta 25.2)$ is at lower field than that of the 3-axial one in $20\left(\delta\right.$ 18.3). Such a behavior is also supportable the above conclusion in accordance with SATo's rule. $\left.{ }^{13}\right)$ Moreover, 3 and 20 have the similar values and signs of specific rotation with those of methyl 3-epi- $\beta$-L-evernitroside (21) $\left(-10.5^{\circ}\left(\mathrm{CHCl}_{3}\right)\right)$ and methyl $\beta$-L-evernitroside $\left.(22)\left(+33.6^{\circ}\left(\mathrm{CHCl}_{3}\right)\right),{ }^{10}\right)$ respectively.

In conclusion the synthesis and the results described herein have provided the satisfactory evidence for the absolute-structure elucidation of decilonitrose (1).

\section{Experimental}

\section{General}

MP's were determined with a Yamato apparatus and are uncorrected. IR spectra were determined on a Hitachi Model 260-10 spectrophotometer. Optical rotations were measured with a Parkin-Elmer 241 polarimeter. The ${ }^{1} \mathrm{H}$ NMR spectra were recorded with Varian XL-100 and Varian EM-390 spectrometers. Chemical shifts are expressed in values (ppm) with TMS as an internal standard. Proton-noise decoupled FT- ${ }^{13} \mathrm{C}$ NMR spectra were taken at $25.2 \mathrm{MHz}$ on a Varian XL-100 spectrometer using TMS as a reference.

Methyl 3-O-Benzoyl-2,6-dideoxy- $\beta$-L-arabino-hexopyranoside (8)

To a solution of $7(2 \mathrm{~g})$ in pyridine $(20 \mathrm{ml})$ was added benzoyl chloride $(1.6 \mathrm{ml})$ at $-50^{\circ} \mathrm{C}$, and the mixture was stirred for 1 hour. After quenching with water, evaporation of the solvent gave an oil, which was subjected to a column chromatography on silica gel. Elution with toluene-ethyl acetate $(20: 1)$ gave an oil of $\mathbf{8}(2.32 \mathrm{~g}, 71 \%)$ : $[\alpha]_{\mathrm{D}}^{24}+26.8^{\circ}\left(c 1.0, \mathrm{CHCl}_{3}\right)$; IR $\left(\mathrm{CHCl}_{3}\right) \mathrm{cm}^{-1} 3450,2970(\mathrm{sh}), 2925,2830(\mathrm{sh})$, $1710,1600,1450 ;{ }^{1} \mathrm{H}$ NMR $\left(90 \mathrm{MHz}, \mathrm{CDCl}_{3}\right) \delta 1.35\left(3 \mathrm{H}, \mathrm{d}, J=5.3 \mathrm{~Hz}, 5-\mathrm{CH}_{3}\right), 1.77\left(1 \mathrm{H}, \mathrm{dt}, J_{2_{\mathrm{ax}}, 1}=9.8 \mathrm{~Hz}\right.$ and $\left.J_{2 \mathrm{ax}, 3} \Rightarrow J_{\mathrm{gem}}=12 \mathrm{~Hz}, 2-\mathrm{H}_{\mathrm{ax}}\right), 2.43\left(1 \mathrm{H}\right.$, ddd, $J_{2 \mathrm{eq}, 1}=2.3 \mathrm{~Hz}, J_{2_{\mathrm{eq}}, 3}=5.3 \mathrm{~Hz}$ and $\left.J_{\mathrm{gem}}=12 \mathrm{~Hz}, 2-\mathrm{H}_{\mathrm{eq}}\right)$, $3.1 \sim 3.8(2 \mathrm{H}, \mathrm{m}, 4-\mathrm{H}$ and $5-\mathrm{H}), 3.50\left(3 \mathrm{H}, \mathrm{s}, 1-\mathrm{OCH}_{3}\right), 4.51\left(1 \mathrm{H}, \mathrm{dd}, J_{1,2_{\mathrm{ax}}}=9.8 \mathrm{~Hz}\right.$ and $J_{1,2_{\text {eq }}}=2.3 \mathrm{~Hz}$, $1-\mathrm{H}), 5.08\left(1 \mathrm{H}\right.$, ddd, $J_{3,2_{\mathrm{eq}}}=5.3 \mathrm{~Hz}, J_{3,2_{\mathrm{ax}}}=12 \mathrm{~Hz}$ and $\left.J_{3,4}=7.8 \mathrm{~Hz}, 3-\mathrm{H}\right), 5.56(1 \mathrm{H}, \mathrm{br} \mathrm{s}, \mathrm{OH}), 7.2 \sim 8.3$ $(5 \mathrm{H}, \mathrm{m}$, phenyl).

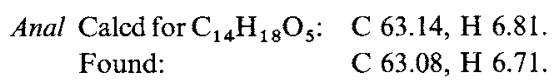


Scheme 1.

$$
\text { (n) }
$$
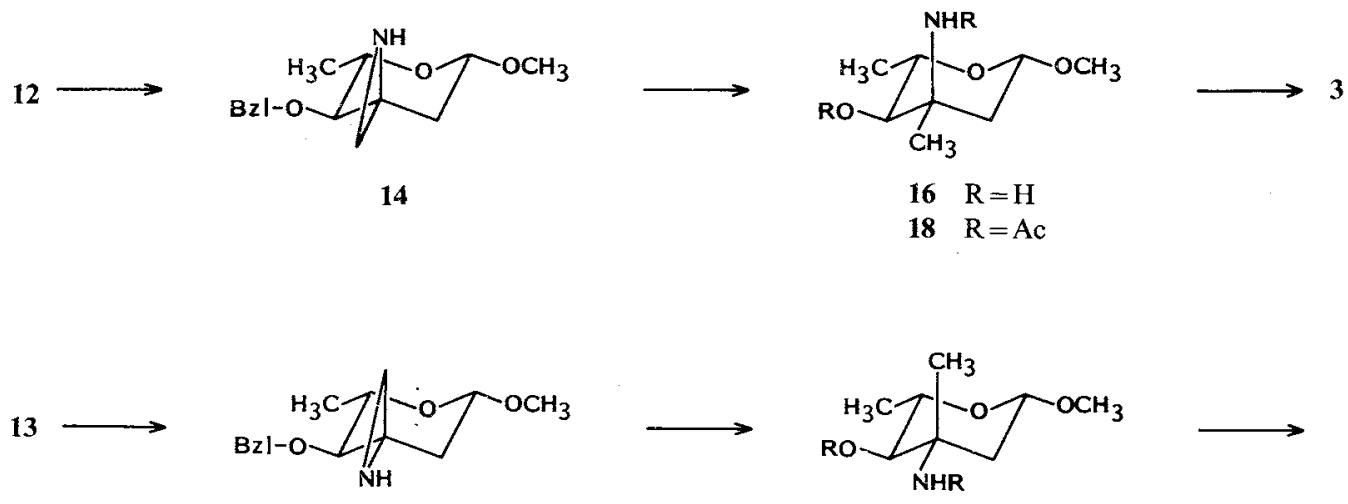
Methyl 3- $O$-Benzoyl-4- $O$-benzyl-2,6-dideoxy- $\beta$-L-arabino-hexopyranoside (9)

After $8(10.5 \mathrm{~g})$ was stirred in DMF $(105 \mathrm{ml})$ with sodium hydride $(1.42 \mathrm{~g})$ at $0^{\circ} \mathrm{C}$ for 30 minutes, benzyl chloride $(7.2 \mathrm{ml})$ was added to the mixture, and the mixture was stirred at room temperature for 1.5 hours. After quenching with water, evaporation of the solvent gave a solid, which was dissolved in chloroform. The chloroform solution was washed with water, dried over $\mathrm{MgSO}_{4}$ and filtered. The filtrate was evaporated to give a foam, which was subjected to a column chromatography on silica gel. Elution with toluene - ethyl acetate $(30: 1)$ gave a colorless solid of $9(9.2 \mathrm{~g}, 64 \%)$ : MP $63 \sim 65^{\circ} \mathrm{C} ;[\alpha]_{\mathrm{D}}^{24}+80.3^{\circ}(c$ $\left.1.0, \mathrm{CHCl}_{3}\right) ; \mathrm{IR}\left(\mathrm{CHCl}_{3}\right) \mathrm{cm}^{-1} 3000(\mathrm{sh}), 2930,2870,2830,1715,1600,1450 ;{ }^{1} \mathrm{H} \mathrm{NMR}\left(90 \mathrm{MHz}, \mathrm{CDCl}_{3}\right)$ $\delta 1.34\left(3 \mathrm{H}, \mathrm{d}, J=5.3 \mathrm{~Hz}, 5-\mathrm{CH}_{3}\right), 1.67\left(1 \mathrm{H}, \mathrm{dt}, J_{2_{\mathrm{ax}}, 1}=9.8 \mathrm{~Hz}\right.$ and $\left.J_{2_{\mathrm{ax}}, 3}=J_{\mathrm{gem}}=12.2 \mathrm{~Hz}, 2-\mathrm{H}_{\mathrm{ax}}\right), 2.45(1 \mathrm{H}$, ddd, $J_{2_{\mathrm{cq}}, 1}=2.3 \mathrm{~Hz}, J_{2_{\mathrm{eq}}, 3}=5.3 \mathrm{~Hz}$ and $\left.J_{\mathrm{gem}}=12.2 \mathrm{~Hz}, 2-\mathrm{H}_{\mathrm{eq}}\right), 3.1 \sim 3.8(2 \mathrm{H}, \mathrm{m}, 4-\mathrm{H}$ and $5-\mathrm{H}), 3.49(3 \mathrm{H}, \mathrm{s}$, $\left.\mathrm{OCH}_{3}\right), 4.44\left(1 \mathrm{H}, \mathrm{dd}, J_{1,2_{\mathrm{eq}}}=2.3 \mathrm{~Hz}\right.$ and $\left.J_{1,2_{\mathrm{ax}}}=9.8 \mathrm{~Hz}, 1-\mathrm{H}\right), 4.60$ and $4.73\left(2 \mathrm{H}, \mathrm{ABq}, J=9.8 \mathrm{~Hz}, \mathrm{CH}_{2}\right.$ of benzyl), $5.30\left(1 \mathrm{H}\right.$, ddd, $J_{3,2_{\mathrm{eq}}}=5.3 \mathrm{~Hz}, J_{3,4}=8.5 \mathrm{~Hz}$ and $\left.J_{3,2 \mathrm{ax}}=12.2 \mathrm{~Hz}, 3-\mathrm{H}\right), 7.2 \sim 8.3(10 \mathrm{H}, \mathrm{m}$, phenyl).

Anal Calcd for $\mathrm{C}_{21} \mathrm{H}_{24} \mathrm{O}_{5}$ : C 70.76, $\mathrm{H} 6.79$.

Found: $\quad$ C $70.48, \mathrm{H} 6.67$.

Methyl 4-O-Benzyl-2,6-dideoxy- $\beta$-L-arabino-hexopyranoside (10)

Compound $9(1.46 \mathrm{~g})$ was dissolved in $1 \%$ methanol solution of sodium methoxide $(140 \mathrm{ml})$, and the mixture was stirred at room temperature for 4 hours. After neutralization with Amberlyst $\mathrm{A}-15\left(\mathrm{H}^{+}\right)$and filtration, the filtrate was evaporated to give a solid, which was dissolved in chloroform $(200 \mathrm{ml})$. The solution was washed with $\mathrm{NaHCO}_{3}$-saturated aqueous solution, dried over $\mathrm{MgSO}_{4}$ and filtered. Evaporation of the filtrate gave a solid, which was crystallized from hexane to afford a colorless crystal of $\mathbf{1 0}$ $(880 \mathrm{mg}, 85 \%)$ : MP $108^{\circ} \mathrm{C} ;[\alpha]_{\mathrm{D}}^{24}+83^{\circ}\left(c 0.2, \mathrm{CHCl}_{3}\right) ; \mathrm{IR}\left(\mathrm{CHCl}_{3}\right) \mathrm{cm}^{-1} 3570(\mathrm{sh}), 2980$ (sh), 2930, 2860 (sh), 2830, 1450, 1390; ${ }^{1} \mathrm{H}$ NMR $\left(90 \mathrm{MHz}, \mathrm{CDCl}_{3}\right) \delta 1.33\left(3 \mathrm{H}, \mathrm{d}, J=6.0 \mathrm{~Hz}, 5-\mathrm{CH}_{3}\right), 1.56(1 \mathrm{H}, \mathrm{dt}$, $J_{2_{\mathrm{ax}, 1}}=9.3 \mathrm{~Hz}$ and $\left.J_{2_{\mathrm{ax}}, 3}=J_{\mathrm{gem}}=12 \mathrm{~Hz}, 2-\mathrm{H}_{\mathrm{ax}}\right), 2.16\left(1 \mathrm{H}, \mathrm{ddd}, J_{2_{\mathrm{eq}}, 1}=2.3 \mathrm{~Hz}, J_{2_{\mathrm{eq}}, 3}=5.3 \mathrm{~Hz}\right.$ and $\left.J_{\mathrm{gem}}=12 \mathrm{~Hz}, 2-\mathrm{H}_{\mathrm{eq}}\right), 2.22(1 \mathrm{H}, \mathrm{d}, J=3.6 \mathrm{~Hz}, \mathrm{OH}), 2.94\left(1 \mathrm{H}, \mathrm{t}, J_{3,4}=J_{4,5}=8.7 \mathrm{~Hz}, 4-\mathrm{H}\right), 3.33(1 \mathrm{H}, \mathrm{dq}$, $J_{5,4}=8.7 \mathrm{~Hz}$ and $\left.J_{5, \mathrm{CH}_{3}}=6.0 \mathrm{~Hz}, 5-\mathrm{H}\right), 3.43\left(3 \mathrm{H}, \mathrm{s}, \mathrm{OCH}_{3}\right), 3.5 \sim 4.0(1 \mathrm{H}, \mathrm{m}, 3-\mathrm{H}), 4.37(1 \mathrm{H}$, dd, $J_{1,2_{\text {eq }}}=2.3 \mathrm{~Hz}$ and $\left.J_{1,2_{\mathrm{ax}}}=9.3 \mathrm{~Hz}, 1-\mathrm{H}\right), 4.72\left(2 \mathrm{H}, \mathrm{s}, \mathrm{CH}_{2}\right.$ of benzyl $), 7.32(5 \mathrm{H}, \mathrm{br}$, phenyl).

Anal Calcd for $\mathrm{C}_{14} \mathrm{H}_{20} \mathrm{O}_{4}$ : C 66.64, $\mathrm{H} 7.99$.

Found:

C $66.50, \mathrm{H} 8.05$.

Methyl 4- $O$-Benzyl-2,6-dideoxy- $\beta$-L-erythro-3-hexulopyranoside (11)

To a solution of chromium(VI) oxide $(3.9 \mathrm{~g})$ in a mixture of dichloromethane $(160 \mathrm{ml})$ and pyridine $(10.8 \mathrm{ml})$ was added $10(0.98 \mathrm{~g})$, and the mixture was stirred at room temperature for 15 minutes. After addition of chloroform and filtration, the filtrate was washed with $\mathrm{NaHCO}_{3}$-saturated aqueous solution and $\mathrm{NaCl}$-saturated aqueous solution, dried over $\mathrm{MgSO}_{4}$ and filtered. Evaporation of the filtrate gave a solid, which was crystallized from hexane to give a colorless crystal of $11(714 \mathrm{mg}, 73 \%)$ : MP $83 \sim 84^{\circ} \mathrm{C}$; $[\alpha]_{\mathrm{D}}^{24}-108^{\circ}\left(c 1.0, \mathrm{CHCl}_{3}\right) ; \mathrm{IR}\left(\mathrm{CHCl}_{3}\right) \mathrm{cm}^{-1} 3000(\mathrm{sh}), 2930,2870,2830,1730,1450,1385,1375,1360$; ${ }^{1} \mathrm{H}$ NMR $\left(90 \mathrm{MHz}, \mathrm{CDCl}_{3}\right) \delta 1.41\left(3 \mathrm{H}, \mathrm{d}, J=5.8 \mathrm{~Hz}, 5-\mathrm{CH}_{3}\right), 2.56\left(1 \mathrm{H}, \mathrm{dd}, J_{2_{\mathrm{ax}, 1}}=8.3 \mathrm{~Hz}\right.$ and $\left.J_{\mathrm{gem}}=13.5 \mathrm{~Hz}, 2-\mathrm{H}_{\mathrm{ax}}\right), 2.78\left(1 \mathrm{H}, \mathrm{dd}, J_{2_{\mathrm{cq}, 1}}=3.8 \mathrm{~Hz}\right.$ and $\left.J_{\mathrm{gem}}=13.5 \mathrm{~Hz}, 2-\mathrm{H}_{\mathrm{eq}}\right), 3.51\left(3 \mathrm{H}, \mathrm{s}, \mathrm{OCH}_{3}\right)$, $3.2 \sim 3.8(2 \mathrm{H}, \mathrm{m}, 4-\mathrm{H}$ and $5-\mathrm{H}), 4.56\left(1 \mathrm{H}, \mathrm{dd}, J_{1,2 \mathrm{ax}}=3.8 \mathrm{~Hz}\right.$ and $\left.J_{1,2 \mathrm{eq}}=8.3 \mathrm{~Hz}, 1-\mathrm{H}\right), 4.47$ and $4.93(2 \mathrm{H}$, ABq, $J=11.2 \mathrm{~Hz}, \mathrm{CH}_{2}$ of benzyl), $7.33(5 \mathrm{H}$, br s, phenyl).

Anal Calcd for $\mathrm{C}_{14} \mathrm{H}_{18} \mathrm{O}_{4}$ : C $67.18, \mathrm{H} 7.25$.

Found:

C 67.16, H 7.03 .

Methyl 4-O-Benzyl-3-C-cyano-2,3,6-trideoxy-3-O-mesyl- $\beta$-L-arabino-hexopyranoside (12) and Methyl 4- $O$-Benzyl-3-C-cyano-2,3,6-trideoxy-3- $O$-mesyl- $\beta$-L-ribo-hexopyranoside (13)

A solution of $11(3.9 \mathrm{~g})$ and an excess of hydrogen cyanide in pyridine $(40 \mathrm{ml})$ was allowed to stand at room temperature overnight. After evaporation, to a solution of the resulting oil in pyridine $(40 \mathrm{ml})$ was added methanesulfonyl chloride $(2.0 \mathrm{ml})$, and the mixture was stirred at room temperature overnight. After quenching with water and evaporation, the resulting oil was dissolved in chloroform, and the solution was washed with water, dried over $\mathrm{MgSO}_{4}$, and filtered. The filtrate was evaporated to give an oil, which was subjected to a column chromatography on silica gel. Elution with hexane - ether $(3: 1)$ gave an oil of $12(2.35 \mathrm{~g}, 42 \%)$ and a colorless solid of $13(1.27 \mathrm{~g}, 22.7 \%) .12:[\alpha]_{\mathrm{D}}^{22}-22.8^{\circ}\left(c 0.5, \mathrm{CH}_{2} \mathrm{Cl}_{2}\right) ; \mathrm{IR}\left(\mathrm{CHCl}_{3}\right)$ 
$\mathrm{cm}^{-1} 1380,1190 ;{ }^{1} \mathrm{H}$ NMR $\left(90 \mathrm{MHz}, \mathrm{CDCl}_{3}\right) \delta 1.28\left(3 \mathrm{H}, \mathrm{d}, J=6.3 \mathrm{~Hz}, 5-\mathrm{CH}_{3}\right), 2.13\left(1 \mathrm{H}, \mathrm{dd}, J_{2_{\mathrm{ax}, 1}}=9.7 \mathrm{~Hz}\right.$ and $\left.J_{\mathrm{gem}}=13.7 \mathrm{~Hz}, 2-\mathrm{H}_{\mathrm{ax}}\right), 2.96\left(3 \mathrm{H}, \mathrm{s}, \mathrm{SO}_{2} \mathrm{CH}_{3}\right), 3.03\left(1 \mathrm{H}, \mathrm{dd}, J_{2_{\mathrm{oq}, 1}}=2.3 \mathrm{~Hz}\right.$ and $\left.J_{\mathrm{gem}}=13.7 \mathrm{~Hz}, 2-\mathrm{H}_{\mathrm{eq}}\right)$, $3.35\left(1 \mathrm{H}, \mathrm{d}, J_{4,5}=9.3 \mathrm{~Hz}, 4-\mathrm{H}\right), 3.45\left(3 \mathrm{H}, \mathrm{s}, \mathrm{OCH}_{3}\right), 3.66\left(1 \mathrm{H}, \mathrm{dq}, J_{5,4}=9.3 \mathrm{~Hz}\right.$ and $\left.J_{5, \mathrm{CH}_{3}}=6.3 \mathrm{~Hz}, 5-\mathrm{H}\right)$, $4.54\left(1 \mathrm{H}, \mathrm{dd}, J_{1,2_{\mathrm{ax}}}=9.7 \mathrm{~Hz}\right.$ and $\left.J_{1,2_{\mathrm{eq}}}=2.3 \mathrm{~Hz}, 2-\mathrm{H}_{\mathrm{eq}}\right), 4.77\left(2 \mathrm{H}, \mathrm{s}, \mathrm{CH}_{2}\right.$ of benzyl), $7.36(5 \mathrm{H}, \mathrm{s}$, phenyl). Anal Calcd for $\mathrm{C}_{16} \mathrm{H}_{21} \mathrm{NO}_{6} \mathrm{~S}$ : C 54.07, H 5.96, N 3.94, S 9.02. Found: C 53.93, H 5.93, N 3.83, S 8.96 .

13: MP $109^{\circ} \mathrm{C}$; $[\alpha]_{\mathrm{D}}^{22}-16.2^{\circ}\left(\mathrm{c} 0.5, \mathrm{CH}_{2} \mathrm{Cl}_{2}\right)$; IR $\left(\mathrm{CHCl}_{3}\right) \mathrm{cm}^{-1} 1380,1190 ;{ }^{1} \mathrm{H}$ NMR $\left(90 \mathrm{MHz}, \mathrm{CDCl}_{3}\right)$ $\delta 1.30\left(3 \mathrm{H}, \mathrm{d}, J=6.0 \mathrm{~Hz}, 5-\mathrm{CH}_{3}\right), 2.04\left(1 \mathrm{H}, \mathrm{dd}, J_{2_{\mathrm{ax}}, 1}=9.3 \mathrm{~Hz}\right.$ and $\left.J_{\mathrm{gem}}=14.5 \mathrm{~Hz}, 2-\mathrm{H}_{\mathrm{ax}}\right), 2.93(1 \mathrm{H}$, dd, $J_{2_{\text {eq }, 1}}=2.3 \mathrm{~Hz}$ and $\left.J_{\mathrm{gem}}=14.5 \mathrm{~Hz}, 2-\mathrm{H}_{\mathrm{eq}}\right), 3.12\left(3 \mathrm{H}, \mathrm{s}, \mathrm{SO}_{2} \mathrm{CH}_{3}\right), 3.47\left(3 \mathrm{H}, \mathrm{s}, \mathrm{OCH}_{3}\right), 3.49(1 \mathrm{H}, \mathrm{d}$, $\left.J_{4,5}=9.3 \mathrm{~Hz}, 4-\mathrm{H}\right), 3.85\left(1 \mathrm{H}, \mathrm{dq}, J_{5,4}=9.3 \mathrm{~Hz}\right.$ and $\left.J_{5, \mathrm{CH}_{3}}=6 \mathrm{~Hz}, 5-\mathrm{H}\right), 4.72\left(1 \mathrm{H}, \mathrm{dd}, J_{1,2_{\text {eq }}}=2.3 \mathrm{~Hz}\right.$ and $\left.J_{1,2_{\mathrm{ax}}}=9.3 \mathrm{~Hz}, 1-\mathrm{H}\right), 4.76$ and $5.03\left(1 \mathrm{H}, \mathrm{ABq}, J=10.5 \mathrm{~Hz}, \mathrm{CH}_{2}\right.$ of benzyl), $7.33(5 \mathrm{H}$, s, phenyl).

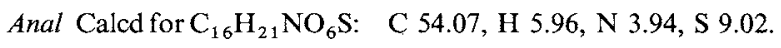

Aziridine-2-spiro-3'-(methyl 4- $O$-Benzyl-2,3,6-trideoxy- $\beta$-L-ribo-hexopyranoside) (14)

To a solution of $12(1.75 \mathrm{~g})$ in ether $(50 \mathrm{ml})$ was added lithium aluminum hydride $(0.56 \mathrm{~g})$, and the mixture was refluxed with stirring. After successive addition of water $(0.5 \mathrm{ml}), 15 \% \mathrm{NaOH}$ aqueous solution $(0.5 \mathrm{ml})$ and water $(0.5 \mathrm{ml})$, the resulting insoluble matter was filtered off and washed with ether. The filtrate and washings were combined and dried over $\mathrm{MgSO}_{4}$, and filtered. Evaporation of the filtrate gave an oil, which was subjected to a column chromatography on silica gel. Elution with toluene - acetone (10:1) gave an oil of $14(862 \mathrm{mg}, 66 \%):[\alpha]_{\mathrm{D}}^{25}+17.6^{\circ}\left(c 1.0, \mathrm{CHCl}_{3}\right)$; IR $\left(\mathrm{CHCl}_{3}\right) \mathrm{cm}^{-1} 3000,2930,2830,1450$, 1385, 1365, 1160, 1075; ${ }^{1} \mathrm{H}$ NMR $\left(90 \mathrm{MHz}, \mathrm{CDCl}_{3}\right) \delta 1.27\left(3 \mathrm{H}, \mathrm{d}, J=6.0 \mathrm{~Hz}, 5-\mathrm{CH}_{3}\right), 1.44(1 \mathrm{H}$, dd,

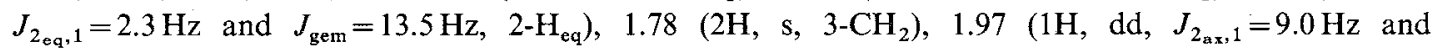
$\left.J_{\mathrm{gem}}=13.5 \mathrm{~Hz}, 2-\mathrm{H}_{\mathrm{ax}}\right), 3.45\left(3 \mathrm{H}, \mathrm{s}, \mathrm{OCH}_{3}\right), 3.2 \sim 3.7(2 \mathrm{H}, \mathrm{m}, 4-\mathrm{H}$ and $5-\mathrm{H}), 4.40$ and $4.60(2 \mathrm{H}, \mathrm{ABq}$, $J=12 \mathrm{~Hz}, \mathrm{CH}_{2}$ of benzyl), $4.67\left(1 \mathrm{H}, \mathrm{dd}, J_{1,2_{\mathrm{eq}}}=2.3 \mathrm{~Hz}\right.$ and $\left.J_{1,2_{\mathrm{ax}}}=9.0 \mathrm{~Hz}, 1-\mathrm{H}\right), 7.30(5 \mathrm{H}$, br s, phenyl).

Anal Caled for $\mathrm{C}_{15} \mathrm{H}_{21} \mathrm{NO}_{3}$ : C 68.41, $\mathrm{H} 8.04, \mathrm{~N} 5.32$. Found:

C 68.23, H 7.88, N 5.20.

Aziridine-2-spiro-3'-(methyl 4-O-Benzyl-2,3,6-trideoxy- $\beta$-L-arabino-hexopyranoside) (15)

Procedures from 13 used were similar to those used for preparation of 14; the yield was $50 \%:[\alpha]_{D}^{24}$ $+47.3^{\circ}\left(c 1.0, \mathrm{CHCl}_{3}\right) ; \mathrm{IR}\left(\mathrm{CHCl}_{3}\right) \mathrm{cm}^{-1} 2975(\mathrm{sh}), 2930,2870,2830,1450,1380,1360,1310,1160,1070$; ${ }^{1} \mathrm{H}$ NMR $\left(90 \mathrm{MHz}, \mathrm{CDCl}_{3}\right) \delta 1.35\left(3 \mathrm{H}, \mathrm{d}, J=6.0 \mathrm{~Hz}, 5-\mathrm{CH}_{3}\right), 1.43\left(1 \mathrm{H}, \mathrm{dd}, J_{2 e q, 1}=2.4 \mathrm{~Hz}\right.$ and $\left.J_{\text {gem }}=13.5 \mathrm{~Hz}, 2-\mathrm{H}_{\mathrm{eq}}\right), 1.95\left(2 \mathrm{H}\right.$, br s, $\left.3-\mathrm{CH}_{2}\right), 2.05\left(1 \mathrm{H}\right.$, ddd, $J_{2_{\mathrm{ax}}, 1}=9.6 \mathrm{~Hz}, J_{\mathrm{gem}}=13.5 \mathrm{~Hz}$ and $\left.J_{2_{\mathrm{ax}, 3-\mathrm{CH}_{2}}}=1.5 \mathrm{~Hz}, 2-\mathrm{H}_{\mathrm{ax}}\right), 3.21\left(1 \mathrm{H}, \mathrm{d}, J_{4,5}=9.6 \mathrm{~Hz}, 4-\mathrm{H}\right), 3.47\left(3 \mathrm{H}, \mathrm{s}, \mathrm{OCH}_{3}\right), 3.2 \sim 3.7(1 \mathrm{H}, \mathrm{m}, 5-\mathrm{H})$, $4.46\left(1 \mathrm{H}, \mathrm{dd}, J_{1,2_{\mathrm{ax}}}=9.6 \mathrm{~Hz}\right.$ and $\left.J_{1,2_{\mathrm{eq}}}=2.4 \mathrm{~Hz}, 1-\mathrm{H}\right), 4.47$ and $4.65\left(2 \mathrm{H}, \mathrm{ABq}, J=9.5 \mathrm{~Hz}, \mathrm{CH}_{2}\right.$ of benzyl), $7.30(5 \mathrm{H}$, br s, phenyl).

$\begin{array}{ll}\text { Anal Calcd for } \mathrm{C}_{15} \mathrm{H}_{21} \mathrm{NO}_{3}: & \mathrm{C} 68.41, \mathrm{H} 8.04, \mathrm{~N} 5.32 . \\ \begin{array}{ll}\text { Found: } & \text { C } 68.15, \mathrm{H} 7.88, \mathrm{~N} 5.18\end{array}\end{array}$

Methyl 3-Amino-2,3,6-trideoxy-3- $C$-methyl- $\beta$-L-ribo-hexopyranoside (16)

A solution of $14(50 \mathrm{mg})$ in methanol $(1.2 \mathrm{ml})$ was stirred under hydrogen at $3.5 \mathrm{~kg} / \mathrm{cm}^{2}$ in the presence of Raney Ni $(250 \mathrm{mg})$ for 18 hours, and then filtered. Evaporation of the filtrate gave an oil, which was subjected to a column chromatography on silica gel. Elution with chloroform - methanol $(5: 1)$ gave an oil of $16(27.6 \mathrm{mg}, 83 \%):[\alpha]_{\mathrm{D}}^{23}+45.6^{\circ}\left(c 1.0, \mathrm{CHCl}_{3}\right) ;[\alpha]_{436}+80^{\circ}\left(c 0.02, \mathrm{H}_{2} \mathrm{O}\right) ;[\alpha]_{436(\mathrm{TACu})}+380^{\circ}(c 0.01$, $\left.\mathrm{H}_{2} \mathrm{O}\right) ; \Delta[\mathrm{M}]_{\mathrm{TACu}}+525^{\circ}$; IR $\left(\mathrm{CHCl}_{3}\right) \mathrm{cm}^{-1} 2960,2930,2840,1450,1380,1320,1165,1135,1075(\mathrm{sh}) ;{ }^{1} \mathrm{H}$ NMR $\left(90 \mathrm{MHz}, \mathrm{CDCl}_{3}\right.$ containing a drop of $\left.\mathrm{D}_{2} \mathrm{O}\right) \delta 1.13\left(3 \mathrm{H}, \mathrm{s}, 3-\mathrm{CH}_{3}\right), 1.27\left(3 \mathrm{H}, \mathrm{d}, J=6.0 \mathrm{~Hz}, 5-\mathrm{CH}_{3}\right)$, $1.55\left(1 \mathrm{H}, \mathrm{dd}, J_{2_{\mathrm{ax}}, 1}=9.0 \mathrm{~Hz}\right.$ and $\left.J_{\mathrm{gem}}=14 \mathrm{~Hz}, 2-\mathrm{H}_{\mathrm{ax}}\right), 1.83\left(1 \mathrm{H}, \mathrm{dd}, J_{2_{\mathrm{eq}}, 1}=3.0 \mathrm{~Hz}\right.$ and $\left.J_{\mathrm{gem}}=14 \mathrm{~Hz}, 2-\mathrm{H}_{\mathrm{eq}}\right)$, $2.90\left(1 \mathrm{H}, \mathrm{d}, J_{4,5}=9.2 \mathrm{~Hz}, 4-\mathrm{H}\right), 3.2 \sim 3.7(1 \mathrm{H}, \mathrm{m}, 5-\mathrm{H}), 3.45\left(3 \mathrm{H}, \mathrm{s}, \mathrm{OCH}_{3}\right), 4.53\left(1 \mathrm{H}, \mathrm{dd}, J_{1,2 \mathrm{H}_{\mathrm{eq}}}=3.0 \mathrm{~Hz}\right.$ and $\left.J_{1,2 \mathrm{H}_{\mathrm{ax}}}=9.0 \mathrm{~Hz}, 1-\mathrm{H}\right)$.

Methyl 3-Amino-2,3,6-trideoxy-3- $C$-methyl- $\beta$-L-arabino-hexopyranoside (17)

Procedures from 15 used were similar to those used for preparation of 16; the yield was $86 \%$ : $[\alpha]_{\mathrm{D}}^{25}$ 
$+33.3^{\circ}\left(c 1.0, \mathrm{CHCl}_{3}\right) ;[\alpha]_{436}+85^{\circ}\left(c 0.02, \mathrm{H}_{2} \mathrm{O}\right) ;[\alpha]_{436(\mathrm{TACu})}-270^{\circ}\left(c 0.01, \mathrm{H}_{2} \mathrm{O}\right) ; \Delta[\mathrm{M}]_{\mathrm{TACu}}-621^{\circ} ; \mathrm{IR}$ $\left(\mathrm{CHCl}_{3}\right) \mathrm{cm}^{-1} 2970,2940,2880,1450,1390,1320,1160,1130,1065 ;{ }^{1} \mathrm{H}$ NMR $\left(90 \mathrm{MHz}, \mathrm{CDCl}_{3}\right) \delta 1.20$ $\left(3 \mathrm{H}, \mathrm{s}, 3-\mathrm{CH}_{3}\right), 1.33\left(3 \mathrm{H}, \mathrm{d}, J=6.0 \mathrm{~Hz}, 5-\mathrm{CH}_{3}\right), 1.59\left(1 \mathrm{H}, \mathrm{dd}, J_{2_{\mathrm{ax}}, \mathrm{1}}=9.7 \mathrm{~Hz}\right.$ and $\left.J_{\mathrm{gem}}=13.0 \mathrm{~Hz}, 2-\mathrm{H}_{\mathrm{ax}}\right)$, $1.95\left(1 \mathrm{H}, \mathrm{dd}, J_{2_{\mathrm{eq}}, 1}=2.3 \mathrm{~Hz}\right.$ and $\left.J_{\mathrm{gem}}=13.0 \mathrm{~Hz}, 2-\mathrm{H}_{\mathrm{eq}}\right), 3.10,\left(1 \mathrm{H}, \mathrm{d}, J_{4,5}=9.8 \mathrm{~Hz}, 4-\mathrm{H}\right), 3.50\left(3 \mathrm{H}, \mathrm{s}, \mathrm{OCH}_{3}\right)$, $3.2 \sim 3.7(1 \mathrm{H}, \mathrm{m}, 5-\mathrm{H}), 4.50\left(1 \mathrm{H}, \mathrm{dd}, J_{1,2_{\mathrm{ax}}}=9.7 \mathrm{~Hz}\right.$ and $\left.J_{1,2_{\mathrm{eq}}}=2.3 \mathrm{~Hz}, 1-\mathrm{H}\right)$.

Methyl 3-Acetamido-4- $O$-acetyl-2,3,6-trideoxy-3- $C$-methyl- $\beta$-L-ribo-hexopyranoside (18)

To a solution of $16(9 \mathrm{mg})$ in pyridine $(0.1 \mathrm{ml})$ was added acetic anhydride $(0.05 \mathrm{ml})$, and the mixture was allowed to stand at room temperature overnight. After quenching with water, evaporation of the solvent gave an oil. The oil was subjected to the preparative TLC of silica gel with toluene - acetone $(3: 1)$ to give an oil of $18(13 \mathrm{mg}, 69 \%)$ : $[\alpha]_{\mathrm{D}}^{23}+11.0^{\circ}\left(c 0.5, \mathrm{CHCl}_{3}\right)$; IR $\left(\mathrm{CHCl}_{3}\right) \mathrm{cm}^{-1} 3430,2975,2930,2870$, $2840,1750,1680,1510,1445 ;{ }^{1} \mathrm{H}$ NMR $\left(90 \mathrm{MHz}, \mathrm{CDCl}_{3}\right) \delta 1.22\left(3 \mathrm{H}, \mathrm{d}, J=6.0 \mathrm{~Hz}, 5-\mathrm{CH}_{3}\right), 1.31(3 \mathrm{H}, \mathrm{s}$, $\left.3-\mathrm{CH}_{3}\right), 1.45\left(2 \mathrm{H}, \mathrm{ddd}, J_{2_{\mathrm{ax}}, 1}=9.5 \mathrm{~Hz}, J_{\mathrm{gem}}=14.3 \mathrm{~Hz}\right.$ and $\left.J_{2_{\mathrm{ax}, \mathrm{NH}}}=1.5 \mathrm{~Hz}, 2-\mathrm{H}_{\mathrm{ax}}\right), 2.03(3 \mathrm{H}, \mathrm{s}, \mathrm{NAc}), 2.16$ $(3 \mathrm{H}, \mathrm{s}, \mathrm{OAc}), 3.43\left(1 \mathrm{H}, \mathrm{dd}, J_{2_{\mathrm{eq}}, 1}=2.2 \mathrm{~Hz}\right.$ and $\left.J_{\mathrm{gem}}=14.3 \mathrm{~Hz}, 2-\mathrm{H}_{\mathrm{eq}}\right), 3.48\left(3 \mathrm{H}, \mathrm{s}, \mathrm{OCH}_{3}\right), 3.78(1 \mathrm{H}, \mathrm{dq}$, $J_{5,4}=9.7 \mathrm{~Hz}$ and $\left.J_{5, \mathrm{CH}_{3}}=6.0 \mathrm{~Hz}, 5-\mathrm{H}\right), 4.54\left(1 \mathrm{H}, \mathrm{dd}, J_{1,2_{\mathrm{ax}}}=9.5 \mathrm{~Hz}\right.$ and $\left.J_{1,2_{\mathrm{eq}}}=2.2 \mathrm{~Hz}, 1-\mathrm{H}\right), 4.63(1 \mathrm{H}, \mathrm{d}$, $\left.J_{4,5}=9.7 \mathrm{~Hz}, 4-\mathrm{H}\right) ;{ }^{13} \mathrm{C}$ NMR $\left(25.2 \mathrm{MHz}, \mathrm{CDCl}_{3}\right) \delta 17.8(\mathrm{q}, \mathrm{C}-6), 20.8$ (q, acetyl $\left.\mathrm{CH}_{3}\right), 24.1$ (q, acetyl $\left.\mathrm{CH}_{3}\right), 24.8\left(\mathrm{q}, 3-\mathrm{CH}_{3}\right), 38.5(\mathrm{t}, \mathrm{C}-2), 56.0(\mathrm{~s}, \mathrm{C}-3), 56.5\left(\mathrm{q}, \mathrm{OCH}_{3}\right), 68.2(\mathrm{~d}, \mathrm{C}-5), 78.0(\mathrm{~d}, \mathrm{C}-4), 99.7(\mathrm{~d}$, $\mathrm{C}-1), 169.3(\mathrm{~s}, \mathrm{C}=\mathrm{O}), 170.2(\mathrm{~s}, \mathrm{C}=\mathrm{O})$.

Anal Calcd for $\mathrm{C}_{12} \mathrm{H}_{21} \mathrm{NO}_{5}$ : C 55.58, H 8.16, N 5.40. Found:

C 55.54, H 7.89, N 5.33 .

Methyl 3-Acetamido-4- $O$-acetyl-2,3,6-trideoxy-3- $C$-methyl- $\beta$-L-arabino-hexopyranoside (19)

Procedures from 17 used were similar to those used for preparation of 18; the yield was $87 \%$ : MP $168^{\circ} \mathrm{C}(\mathrm{dec}) ;[\alpha]_{\mathrm{D}}^{23}+12.4^{\circ}\left(c 1.0, \mathrm{CHCl}_{3}\right) ; \mathrm{IR}\left(\mathrm{CHCl}_{3}\right) \mathrm{cm}^{-1} 3430,2980,2930,2875,2830,1720,1680$, 1520,$1450 ;{ }^{1} \mathrm{H}$ NMR $\left(90 \mathrm{MHz}, \mathrm{CDCl}_{3}\right) \delta 1.22\left(3 \mathrm{H}, \mathrm{d}, J=6.0 \mathrm{~Hz}, 5-\mathrm{CH}_{3}\right), 1.53\left(3 \mathrm{H}, \mathrm{s}, 3-\mathrm{CH}_{3}\right), 1.78(1 \mathrm{H}$, $\mathrm{dd}, J_{2_{\mathrm{ax}}, 1}=9.8 \mathrm{~Hz}$ and $\left.J_{\mathrm{gem}}=13.5 \mathrm{~Hz}, 2-\mathrm{H}_{\mathrm{ax}}\right), 1.87(3 \mathrm{H}, \mathrm{s}, \mathrm{NAc}), 2.16(3 \mathrm{H}, \mathrm{s}, \mathrm{OAc}), 2.75(1 \mathrm{H}$, dd, $J_{2_{\mathrm{eq}}, 1}=2.3 \mathrm{~Hz}$ and $\left.J_{\mathrm{gem}}=13.5 \mathrm{~Hz}, 2-\mathrm{H}_{\mathrm{eq}}\right), 3.46\left(3 \mathrm{H}, \mathrm{s}, \mathrm{OCH}_{3}\right), 3.72\left(1 \mathrm{H}, \mathrm{dq}, J_{5,4}=9.7 \mathrm{~Hz}\right.$ and $J_{5, \mathrm{CH}_{3}}=6.0 \mathrm{~Hz}$, $5-\mathrm{H}), 4.49\left(1 \mathrm{H}, \mathrm{dd}, J_{1,2_{\mathrm{ax}}}=9.8 \mathrm{~Hz}\right.$ and $\left.J_{1,2_{\mathrm{eq}}}=2.3 \mathrm{~Hz}, 1-\mathrm{H}\right), 4.67\left(1 \mathrm{H}, \mathrm{d}, J_{4,5}=9.7 \mathrm{~Hz}, 4-\mathrm{H}\right) ;{ }^{13} \mathrm{C} \mathrm{NMR}$ $\left(25.2 \mathrm{MHz}, \mathrm{CDCl}_{3}\right) \delta 17.8(\mathrm{q}, \mathrm{C}-6), 18.3\left(\mathrm{q}, 3-\mathrm{CH}_{3}\right), 21.0\left(\mathrm{q}\right.$, acetyl $\left.\mathrm{CH}_{3}\right), 24.5\left(\mathrm{q}\right.$, acetyl $\left.\mathrm{CH}_{3}\right), 41.9(\mathrm{t}$, $\mathrm{C}-2), 56.3(\mathrm{~s}, \mathrm{C}-3), 56.5\left(\mathrm{q}, \mathrm{OCH}_{3}\right), 68.1(\mathrm{~d}, \mathrm{C}-5), 78.3(\mathrm{~d}, \mathrm{C}-4), 99.7(\mathrm{~d}, \mathrm{C}-1), 169.5(\mathrm{~s}, \mathrm{C}=\mathrm{O}), 172.3(\mathrm{~s}, \mathrm{C}=\mathrm{O})$.

Anal Calcd for $\mathrm{C}_{12} \mathrm{H}_{21} \mathrm{NO}_{5}:$ C $55.58, \mathrm{H} 8.16, \mathrm{~N} 5.40$.

Found:

C 55.52, H 7.94, N 5.21 .

Methyl 2,3,6-Trideoxy-3- $C$-methyl-3-nitro- $\beta$-L-ribo-hexopyranoside (Methyl $\beta$-Decilonitroside, 3)

To a solution of $16(40 \mathrm{mg})$ in acetonitrile $(2 \mathrm{ml})$ was added dropwise a solution of $m$-chloroperbenzoic acid $(120 \mathrm{mg})$ in acetonitrile $(8 \mathrm{ml})$, and the mixture was stirred at $25^{\circ} \mathrm{C}$ for 25 minutes. After quenching with $10 \%$ sodium thiosulfate aqueous solution and extraction with dichloromethane, the extract was washed with $\mathrm{NaHCO}_{3}$-saturated aqueous solution and water, dried over $\mathrm{MgSO}_{4}$, and filtered. Evaporation of the filtrate gave an oil, which was subjected to the preparative TLC of silica gel with dichloromethane to give a foam of $\mathbf{3}\left(13.8 \mathrm{mg}, 29 \%\right.$, easily sublimes under a reduced pressure): $[\alpha]_{\mathrm{D}}^{25}-12.0^{\circ}\left(\mathrm{c} 0.2, \mathrm{CHCl}_{3}\right)$ (natural 3, $-13^{\circ}\left(c 0.2, \mathrm{CHCl}_{3}\right)^{2}$; IR $\left(\mathrm{CHCl}_{3}\right) \mathrm{cm}^{-1} 2975,2925,2840,1540,1445,1425,1400,1380,1350$, $1315,1285,1160,1125,1075 ;{ }^{1} \mathrm{H}$ NMR $\left(90 \mathrm{MHz}, \mathrm{CDCl}_{3}\right) \delta 1.37\left(3 \mathrm{H}, \mathrm{d}, J=6.0 \mathrm{~Hz}, 5-\mathrm{CH}_{3}\right), 1.72(3 \mathrm{H}, \mathrm{s}$, $\left.3-\mathrm{CH}_{3}\right), 1.77\left(1 \mathrm{H}, \mathrm{dd}, J_{2_{\mathrm{ax}}, 1}=9.4 \mathrm{~Hz}\right.$ and $\left.J_{\mathrm{gem}}=14.7 \mathrm{~Hz}, 2-\mathrm{H}_{\mathrm{ax}}\right), 2.73\left(1 \mathrm{H}, \mathrm{dd}, J_{2_{\mathrm{eq}}, 1}=2.0 \mathrm{~Hz}\right.$ and $\left.J_{\text {gem }}=14.7 \mathrm{~Hz}, 2-\mathrm{H}_{\mathrm{eq}}\right), 2.9 \sim 3.5(2 \mathrm{H}, \mathrm{m}, 4-\mathrm{H}$ and $4-\mathrm{OH}), 3.48\left(3 \mathrm{H}, \mathrm{s}, \mathrm{OCH}_{3}\right), 3.70\left(1 \mathrm{H}, \mathrm{dq}, J_{5,4}=9.0 \mathrm{~Hz}\right.$ and $\left.J_{5, \mathrm{CH}_{3}}=6.0 \mathrm{~Hz}, 5-\mathrm{H}\right), 4.50\left(1 \mathrm{H}, \mathrm{dd}, J_{1,2_{\mathrm{ax}}}=9.4 \mathrm{~Hz}\right.$ and $\left.J_{1,2_{\mathrm{eq}}}=2.0 \mathrm{~Hz}, 1-\mathrm{H}\right) ;{ }^{13} \mathrm{C} \mathrm{NMR}(25.2 \mathrm{MHz}$, $\mathrm{CDCl}_{3}$ ) $\delta 18.3(\mathrm{q}, \mathrm{C}-6), 25.2\left(\mathrm{q}, 3-\mathrm{CH}_{3}\right), 41.7(\mathrm{t}, \mathrm{C}-2), 56.5\left(\mathrm{q}, \mathrm{OCH}_{3}\right), 71.1$ (d, C-5), 77.0 (d, C-4), 89.6 (s, C-3), 98.6 (d, C-1).

Methyl 2,3,6-Trideoxy-3- $C$-methyl-3-nitro- $\beta$-L-arabino-hexopyranoside (20)

Procedures from 17 used were similar to those used for preparation of 3 ; the yield was $62 \%:[\alpha]_{\mathbb{D}}^{25}$ $+41.7^{\circ}\left(\mathrm{c} \mathrm{1.0}, \mathrm{CHCl}_{3}\right) ; \mathrm{IR}\left(\mathrm{CHCl}_{3}\right) \mathrm{cm}^{-1} 3580,3370(\mathrm{sh}), 2970,2940,2870,2830,1540,1450,1390,1365$, $1350,1320,1165,1120 ;{ }^{1} \mathrm{H}$ NMR $\left(90 \mathrm{MHz}, \mathrm{CDCl}_{3}\right) \delta 1.38\left(3 \mathrm{H}, \mathrm{d}, J=6.0 \mathrm{~Hz}, 5-\mathrm{CH}_{3}\right), 1.69\left(3 \mathrm{H}, \mathrm{s}, 3-\mathrm{CH}_{3}\right)$, $2.12\left(1 \mathrm{H}, \mathrm{dd}, J_{2_{\mathrm{ax}}, 1}=9.0 \mathrm{~Hz}\right.$ and $\left.J_{\mathrm{gem}}=13.5 \mathrm{~Hz}, 2-\mathrm{H}_{\mathrm{ax}}\right), 2.38\left(1 \mathrm{H}, \mathrm{dd}, J_{2_{\mathrm{eq}}, 1}=2.3 \mathrm{~Hz}\right.$ and $J_{\mathrm{gem}}=13.5 \mathrm{~Hz}$, 
2- $\left.\mathrm{H}_{\mathrm{eq}}\right), 3.0(1 \mathrm{H}$, br s, $4-\mathrm{OH}), 3.45\left(1 \mathrm{H}, \mathrm{dq}, J_{5,4}=9.0 \mathrm{~Hz}\right.$ and $\left.J_{5, \mathrm{CH}_{3}}=6.0 \mathrm{~Hz}, 5-\mathrm{H}\right), 3.48\left(3 \mathrm{H}, \mathrm{s}, \mathrm{OCH}_{3}\right)$, $3.94\left(1 \mathrm{H}\right.$, br d, $\left.J_{4,5}=9.0 \mathrm{~Hz}, 4-\mathrm{H}\right), 4.48\left(1 \mathrm{H}, \mathrm{dd}, J_{1,2_{\mathrm{eq}}}=2.3 \mathrm{~Hz}\right.$ and $\left.J_{1,2 \mathrm{ax}}=9.0 \mathrm{~Hz}, 1-\mathrm{H}\right) ;{ }^{13} \mathrm{C} \mathrm{NMR}$ $\left(25.2 \mathrm{MHz}, \mathrm{CDCl}_{3}\right), 18.1$ (q, 3-CH $\mathrm{CH}_{3}$ or $\left.\mathrm{C}-6\right), 18.3\left(\mathrm{q}, \mathrm{C}-6\right.$ or $\left.3-\mathrm{CH}_{3}\right), 41.4(\mathrm{t}, \mathrm{C}-2), 56.5\left(\mathrm{q}, \mathrm{OCH}_{3}\right), 70.5$ (d, C-5), 74.6 (d, C-4), 89.3 (s, C-3), 99.1 (d, C-1).

\section{References}

1) Ishit, K.; S. Kondo, Y. Nishimura, M. Hamada, T. Takeuchi \& H. Umezawa: Decilorubicin, a new anthracycline antibiotic. J. Antibiotics 36: $451 \sim 453,1983$

2) Ishil, K; Y. Nishimura, S. Kondo \& H. UmeZAWA: Decilonitrose and 4- $O$-succinyl-L-diginose, sugar components of decilorubicin. J. Antibiotics 36: 454 456, 1983

3) Kawai, H.; Y. Hayakawa, M. Nakagawa, K. Furihata, H. Seto \& N. Ōtake: The structure of arugomycin, a new anthracycline antibiotic. Part I. Structural elucidation of degradation products, AG1, AG2 and AG3. Tetrahedron Lett. 25: 1937 1940, 1984

4) Kawal, H.; Y. Hayakawa, M. Nakagawa, K. Furihata, H. Seto \& N. Ōtake: Arugomycin, a new anthracycline antibiotic. II. Structural elucidation. J. Antibiotics 40: 1273 1282, 1987

5) Kind, R.; K. HÜtTeR, A. ZeECK, K. SChMidT-BÄSE \& E. EgerT: Viriplanin A, a new anthracycline antibiotic of the nogalamycin group. II. The structure of a novel hydroxyamino sugar from reduced viriplanin A. J. Antibiotics 42: $7 \sim 13,1989$

6) Hudson, C. S.: The classification of anomers by the symbols $\alpha-\mathrm{D}, \alpha-\mathrm{L}, \beta$-D and $\beta$-L. Adv. Carbohydr. Chem. 3: $15 \sim 18,1948$

7) Brimacombe, J. S. \& K. M. M. Raiman: The synthesis of a derivative of L-decilonitrose (2,3,6-trideoxy-3-C-methyl3-nitro-L-ribo-hexose). Carbohydr. Res. 140: 163 166, 1985

8) Ishil, K.; Y. Nishimura, S. Kondo \& H. UmEZAWA: The synthesis of sugar components of decilorubicin. Abstracts Papers of Annual Meeting of the Agricultural Chemical Society of Japan, No. 3I-21, p. 462, Sendai, Mar. $29 \sim$ Apr. 1, 1983

9) Ishit, K.: Chemical studies on a new antitumor antibiotic, decilorubicin. Ph. D. Thesis, Univ. Tokyo, 1983

10) Yoshimura, J.; M. Matsuzawa, K. Sato \& Y. Nagasawa: The synthesis of evernitrose and 3-epi-evernitrose. Carbohydr. Res. 76: $67 \sim 78,1979$

11) Allgeier, H.: Struktur der Pachybiose und Asclepobiose. Helv. Chim. Acta 51: 311 325, 1968

12) Umezawa, S.; T. Tsuchrya \& K. TAtsuta: Studies of aminosugars. XI. Configurational studies of aminosugar glycosides and aminocyclitols by a copper complex method. Bull. Chem. Soc. Jpn. 39: 1235 1243, 1966

13) Sato, K.; M. Matsuzawa, K. Ajisaka \& J. Yoshimura: Branched-chain sugars. XIX. On the application of ${ }^{13} \mathrm{C}$ NMR spectroscopy to the configurational assignment of 3-C-substituents of aldohexopyranose derivatives. Bull. Chem. Soc. Jpn. 53: 189 191, 1980 